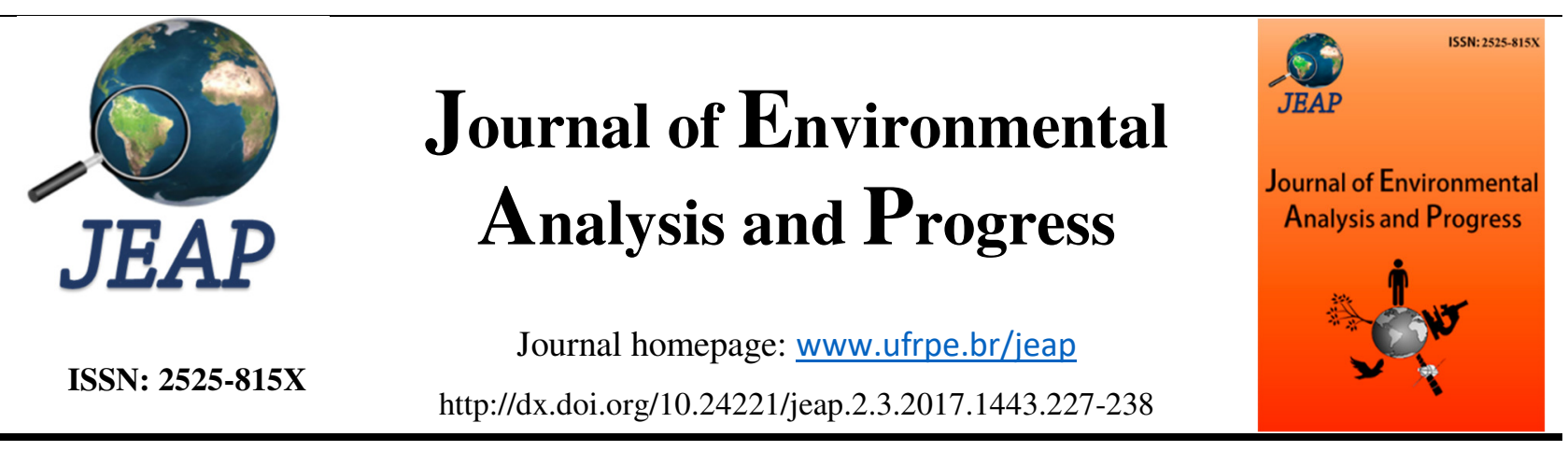

\title{
Modelagem hidrológica sob escassez de dados na Bacia do Alto Mundaú, Nordeste do Brasil
}

\section{Hydrological modeling on data scarcity in the Upper Mundaú Basin, Northeast of Brazil}

Carolyne Wanessa Lins de Andrade ${ }^{\mathrm{a}}$, Suzana Maria Gico Lima Montenegro ${ }^{\mathrm{b}}$, José Romualdo de Sousa Lima ${ }^{\mathrm{c}}$, Abelardo Antônio de Assunção Montenegro ${ }^{\mathrm{a}}$, Adriana Guedes Magalhães ${ }^{\mathrm{d}}$

${ }^{a}$ Universidade Federal Rural de Pernambuco-UFRPE, Rua Dom Manoel de Medeiros, s/n, Dois Irmãos, RecifePernambuco, Brasil. CEP: 52171-900. E-mail: carolyne.andrade@ufrpe.br; abelardo.montenegro@ufrpe.br.

${ }^{\mathrm{b}}$ Universidade Federal de Pernambuco-UFPE, Av. Prof. Moraes Rego, 1235, Cidade Universitária, Recife, Pernambuco, Brasil. CEP: 50670-901. E-mail: suzanam@ufpe.br.

${ }^{\mathrm{c}}$ UFRPE, Unidade Acadêmica de Garanhuns-UAG, Av. Bom Pastor, s/n, Boa Vista, Garanhuns/PE, Brasil. CEP: $55292-$ 270. E-mail: romualdo.lima@ufrpe.br.

d Faculdade Santa Helena-FSH, Av. Caxangá, n. 990, Madalena, Recife-PE, Brasil. CEP: 50711-000. E-mail: agmguedes@gmail.com.

\section{A R T I C L E I N F O}

Recebido 28 Jun 2017

Aceito 10 Jul 2017

Publicado 31 Jul 2017

\begin{abstract}
A B S T R A C T
Studies focused on the understanding of hydrological processes are of paramount importance to assist in the management and decision-making related to water resources in watersheds. Hydrological models are considered powerful tools in understanding these processes. However, such models require a range of data that is not easily accessible. This study aimed to apply and test the SWAT model, following a recommended sequence of procedures in a sub-basin of the Mundaú River, in the Northeast of Brazil. Input data were used as a land use map, a digital elevation map and a soil type map, as well as a period data of 2000 to 2016, in daily time step. The accuracy of the model based on the Nash-Sutcliffe coefficient (CNS), by percentage of trend (PBIAS) and by the coefficient of determination $\left(\mathrm{R}^{2}\right)$. The values of CNS, PBIAS and $\mathrm{R}^{2}$ were $0.53 ;-7.1$ and 0.56 , respectively, for the calibration period. It was possible to perform the hydrological modeling with the SWAT model in the Mundaú river sub-basin, obtaining the satisfactory performance, according to the CNS and very good considering the PBIAS in the calibration. With the calibrated model, future studies can be carried out with the SWAT to validate it and predict possible impacts of climate change and land use in the studied basin. Studies such as this have shown that, increasingly, experimental data are essential for adequate representation and understanding of processes through hydrological modeling.
\end{abstract}

Keywords: SWAT model, calibration, flow.

\section{R E S U M O}

Estudos voltados ao entendimento dos processos hidrológicos são de suma importância para auxiliar a gestão e a tomada de decisões relacionadas aos recursos hídricos. Modelos hidrológicos são considerados ferramentas poderosas no entendimento desses processos. Entretanto, tais modelos requerem uma série de dados que não estão facilmente acessíveis. Este estudo objetivou aplicar e testar o modelo SWAT, seguindo uma sequência de procedimentos recomendada em uma sub-bacia do rio Mundaú, Nordeste do Brasil. Foram utilizados dados de entrada como mapa de uso e ocupação do solo, mapa digital de elevação e mapa dos tipos de solo, além de um período de 2000 a 2016, em passo de tempo diário. A acurácia do 
modelo foi medida com base no coeficiente de Nash-Sutcliffe (CNS), pelo percentual de tendência (PBIAS) e pelo coeficiente de determinação $\left(\mathrm{R}^{2}\right)$. Os valores de CNS, PBIAS e $\mathrm{R}^{2}$ obtidos foram de 0,$53 ;-7,1$ e 0,56 respectivamente, para o período de calibração. Foi possível realizar a modelagem hidrológica com o modelo SWAT na sub-bacia do rio Mundaú, obtendo-se desempenho satisfatório, segundo o CNS e muito bom considerando o PBIAS na calibração. Com o modelo calibrado, estudos futuros podem ser realizados com o SWAT, a fim de validá-lo e prever possíveis impactos de mudanças climáticas e de uso do solo na bacia estudada. Estudos como este têm mostrado que cada vez mais, os dados experimentais são imprescindíveis para a adequada representação e entendimento dos processos por meio da modelagem hidrológica.

Palavras-Chave: Modelo SWAT, calibração, vazão.

\section{Introdução}

O aumento dos padrões de vida do homem, o crescimento populacional e o manejo inadequado dos recursos naturais, entre outros fatores, estão agravando os problemas relacionados ao abastecimento local, regional e nacional de água, utilizado principalmente para fins como a irrigação, produção de energia, industriais, domésticos e o meio ambiente (Abbaspour et al., 2015). Em regiões com variações interanuais de precipitações marcantes e altas taxas evaporativas, como a do semiárido do Brasil, os problemas de abastecimento são ainda maiores. Assim, estudos voltados ao entendimento dos processos hidrológicos são de suma importância para auxiliar a gestão e a tomada de decisões. Segundo Montenegro et al. (2014) o conhecimento das variáveis hidrológicas representa um aspecto importante para o manejo dos recursos hídricos.

Diversas ferramentas de suporte à tomada de decisões são necessárias para subsidiar a implementação de políticas e auxiliar no manejo dos recursos naturais brasileiros. Segundo Abbaspour et al. (2015) os modelos hidrológicos são importantes para o planejamento dos recursos hídricos no atendimento às diversas demandas, auxiliando no seu uso sustentável. A maior ou menor capacidade que um modelo hidrológico possui em representar a realidade dos processos, depende, em grande parte, da confiabilidade dos dados experimentais. Desta forma, a inclusão de dados "soft" e "hard" que apresentem alta qualidade e confiabilidade é primordial para a obtenção de resultados satisfatórios. Dados "soft" incluem informações sobre o conhecimento qualitativo dos modeladores ou a partir da literatura científica, como relatórios, teses e dissertações. Como exemplo desses dados, tem-se evapotranspiração, o escoamento de base, a profundidade dos aquíferos, índice de área foliar e produtividade. Dados "hard" são os dados medidos, como vazão, níveis de lençóis freáticos, umidade do solo. Esses dados são geralmente utilizados na calibração (Bressiani, 2016). Entretanto, a obtenção de dados de boa qualidade em países em desenvolvimento, como o Brasil, não é uma tarefa fácil. As estações de monitoramento, em muitas regiões brasileiras, não estão em quantidade, distribuição e tempo de operação suficientes ou adequados (Bressiani et al., 2015), configurando os problemas relacionados à escassez de dados.

Deste modo, este estudo objetivou aplicar e testar o modelo SWAT, seguindo uma sequência de procedimentos recomendada em uma sub-bacia do rio Mundaú, Nordeste do Brasil, buscando resultados relacionados à viabilidade do uso do modelo na região.

\section{Material e Métodos \\ Caracterização da área de estudo}

$\mathrm{O}$ estudo foi desenvolvido em uma subbacia $\left(754,99 \mathrm{~km}^{2}\right)$ da Bacia Hidrográfica do Rio Mundaú-BHRM (4.126 km²), Brasil, localizada entre as coordenadas $08^{\circ} 41^{\prime} 34^{\prime \prime}$ e $09^{\circ} 14^{\prime} 00^{\prime \prime}$ de latitude sul, e $36^{\circ} 03^{\prime} 36^{\prime \prime}$ e $36^{\circ} 37^{\prime} 27^{\prime \prime}$ de longitude oeste (Figura 1). A região apresenta clima, segundo a classificação de Köppen, Aw (Tropical, com estação seca no inverno), sendo uma pequena porção a noroeste da bacia composta pelo clima BSh (clima das estepes quentes com baixas latitudes e altitudes) (Pauwels, 2002). A maior parte da bacia hidrográfica do rio Mundaú é classificada em relação a umidade como semiúmida (4 a 5 meses de seca), e uma pequena porção classificada como semiárida ( 7 a 8 meses de seca), caracterizada também por temperaturas quentes (médias acima de $18^{\circ} \mathrm{C}$ todos os meses) (Quadro et al., 1996). A precipitação média anual na BHRM é de $900 \mathrm{~mm}$. Na faixa litorânea encontram-se os valores pluviométricos máximos, com médias anuais em torno de $2.000 \mathrm{~mm}$. À medida que se adentra no continente, há uma diminuição pluviométrica devido à aproximação da região do polígono das secas.

$\mathrm{Na}$ BHRM são encontrados os biomas Caatinga, caracterizado por arbustos e florestas sazonalmente secas (Leal et al., 2005) e Mata Atlântica, caracterizada por florestas sazonalmente secas ou tropicais (Werneck, 2011). São 
encontradas também restingas e manguezais; de vegetações originais da Caatinga, Floresta Tropical Atlântica e vegetação litorânea.

A bacia do rio Mundaú possui grande relevância para a atividade agropecuária, onde é considerada, na porção pernambucana, como uma bacia leiteira, devido às grandes produções de laticínios advindas da criação de bovinos, além da criação de frangos de corte. Também possui grande importância para o cultivo da cana-de-açúcar na porção alagoana, uma vez que nessas áreas estão concentradas grandes usinas de produção de álcool e açúcar. A bacia é predominantemente rural e coberta principalmente por pastagem e cana-deaçúcar, mas ainda possui áreas com cobertura florestal e áreas urbanas.
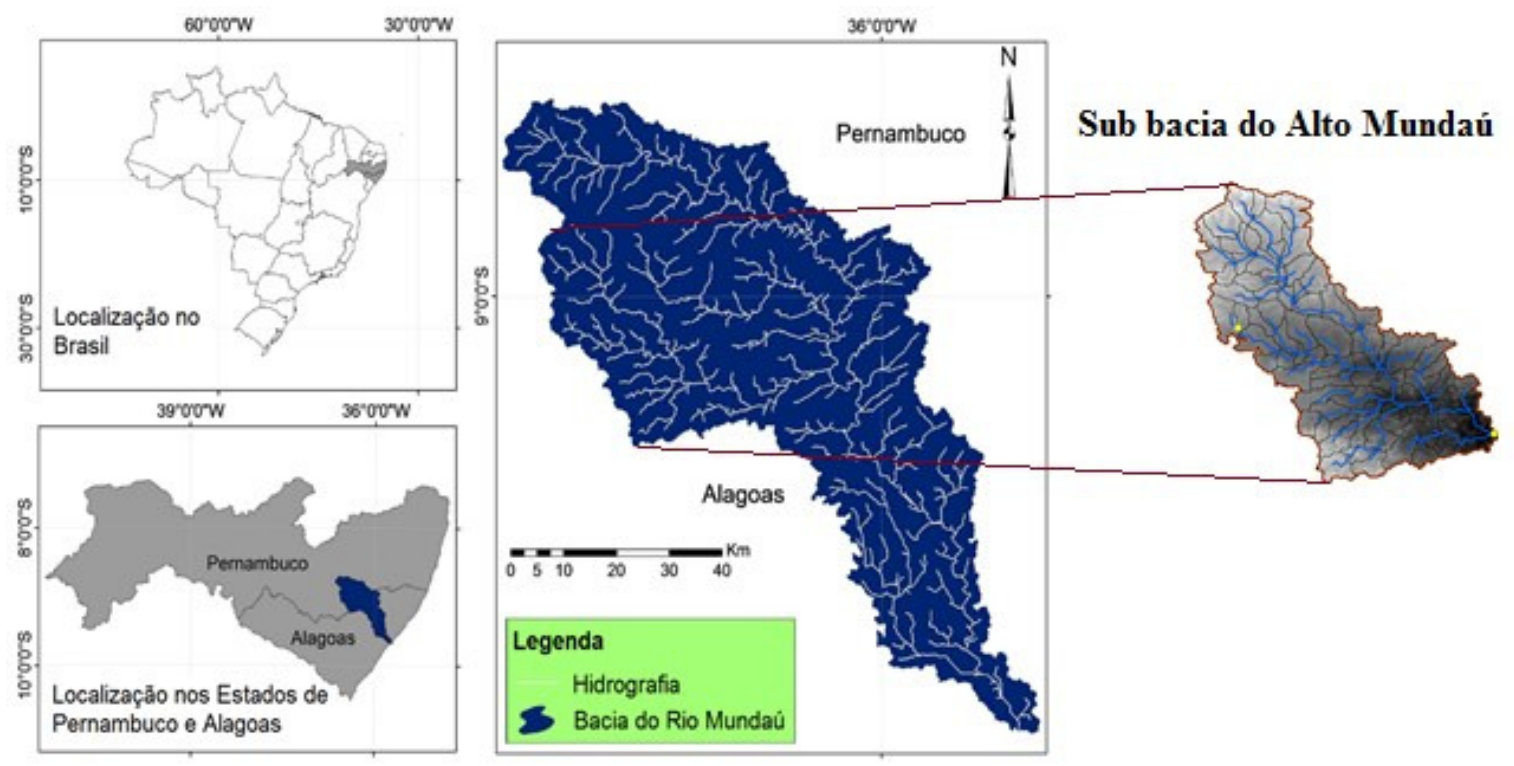

Figura 1. Localização da sub-bacia da Bacia Hidrográfica do Rio Mundaú-BHRM e da sub-bacia pertencente ao Alto Mundaú.

\section{Modelo SWAT}

O SWAT (Soil and Water Assessment Tool), disponibilizado livremente em http://swat.tamu.edu, é um modelo semi conceitual, semi distribuído, de base física e contínuo no tempo, o que permite que diferentes processos físicos sejam simulados na área de estudo. Os principais componentes do modelo são: clima, hidrologia (escoamento superficial, percolação, interceptação, infiltração, escoamento subsuperficial, escoamento de base e evapotranspiração), temperatura do solo, crescimento de plantas, nutrientes, pesticidas, bactérias e patógenos, e manejo do solo (Arnold et al., 2012).

O ciclo hidrológico no SWAT é baseado na Equação do balanço hídrico:

$$
S W_{t}=S W_{0}+\sum_{i=1}^{t}\left(P-Q_{s}-E T-W_{s}-Q_{g w}\right)
$$

onde $\mathrm{SW}_{\mathrm{t}}$ e $\mathrm{SW}_{0}$ são armazenamento de água no solo nos tempos final e inicial, respectivamente $(\mathrm{mm}), \mathrm{t}$ é tempo (dias), P é precipitação $(\mathrm{mm}), \mathrm{Q}_{\mathrm{s}}$ é escoamento superficial (mm), ET é evapotranspiração $(\mathrm{mm}), \mathrm{W}_{\mathrm{s}}$ é percolação $(\mathrm{mm})$ e
$\mathrm{Q}_{\mathrm{gw}}$ é o escoamento de base (mm) (Neitsch et al., 2005).

O escoamento superficial é simulado com o modelo empírico da curva-número (método padrão) do Soil Conservation Service (SCS), que se constitui em uma base única para estimar o escoamento sob diferentes condições de uso e ocupação do solo (Rallison \& Miller, 1981).

$Q_{S}=\frac{\left(P-I_{a}\right)^{2}}{P-I_{a}+S}$

onde P é precipitação (mm), $\mathrm{I}_{\mathrm{a}}$ é a abstração inicial que inclui armazenamento superficial, interceptação e infiltração antes do escoamento $(\mathrm{mm})$ comumente aproximada para 0,2 no numerador e 0,8 no denominador; e $\mathrm{S}$ é o parâmetro de retenção da água $(\mathrm{mm})$, que varia espacialmente de acordo com o tipo solo, cobertura vegetal, manejo e declividade, e CN é a curva número (Eq. $3)$.

$$
S=25,4\left(\frac{1000}{C N}-10\right)
$$

A vazão de pico, ou seja, a vazão máxima de escoamento superficial que ocorre em determinado evento de chuva, é calculada pelo 
modelo SWAT a partir do método racional. O método racional considera que uma determinada chuva com intensidade i começando no tempo t, terá a sua taxa de escoamento aumentada até atingir o tempo de concentração $t_{\text {conc. }}$ O método é dado pela Equação 4 (Neitsch et al., 2005).

$q_{\text {peak }}=\frac{C * i * A}{3,6}$

onde $q_{\text {peak }}$ é a vazão de pico $\left(\mathrm{m}^{3} \mathrm{~s}^{-1}\right)$, $\mathrm{C}$ é o coeficiente de escoamento superficial, i é a intensidade de precipitação $\left(\mathrm{mm} \mathrm{h}^{-1}\right)$ e $\mathrm{A}$ é a área da bacia $\left(\mathrm{km}^{2}\right)$.

O modelo SWAT disponibiliza três opções para a estimativa da componente evapotranspiração potencial $\left(\mathrm{ET}_{0}\right)$ : PenmanMonteith-FAO, Hargreaves \& Samani (1985) e Priestley-Taylor. O método padrão do modelo é o Penman-Monteith-FAO, que requer variáveis meteorológicas como radiação solar, temperatura, umidade relativa e velocidade do vento para a estimativa da $\mathrm{ET}_{0}$ (Neitsch et al., 2005). No presente estudo foi utilizado o método de PenmanMonteith-FAO, devido a disponibilidade de dados agroclimatológicos na parte alta da bacia. A equação é dada por:

$E T_{0}=\frac{0,408 \Delta\left(R_{n}-G\right)+\gamma \frac{900}{T+273} v_{2}\left(e_{s}-e_{a}\right)}{\Delta+\gamma\left(1+0,34 v_{2}\right)}(5)$

onde $\mathrm{ET}_{0}$ é evapotranspiração potencial (mm.dia $\left.{ }^{-1}\right)$, $\Delta$ declividade da curva da pressão de saturação de vapor com a temperatura $\left(\mathrm{kPa} \cdot{ }^{\circ} \mathrm{C}^{-1}\right), \mathrm{R}_{\mathrm{n}}$ saldo de radiação $\left(\mathrm{MJ} \mathrm{m}^{-2} \cdot \operatorname{dia}^{-1}\right), \mathrm{G}$ densidade de fluxo de calor do solo $\left(\mathrm{MJ}^{-2} \mathrm{~m}^{-2} \cdot \mathrm{dia}^{-1}\right), \quad \gamma$ constante psicrométrica $\left(\mathrm{kPa} .{ }^{\circ} \mathrm{C}^{-1}\right), \mathbf{v}_{2}$ velocidade do vento $(\mathrm{m}$ $\left.\mathrm{s}^{-1}\right), \mathrm{e}_{\mathrm{s}}$ pressão de saturação do vapor d'água $(\mathrm{kPa})$, $\mathrm{e}_{\mathrm{a}}$ pressão atual de vapor d'água $(\mathrm{kPa})$ e $\mathrm{T}$ temperatura média do ar $\left({ }^{\circ} \mathrm{C}\right)$.

\section{Modelagem hidrológica com o modelo SWAT na sub-bacia da BHRM}

A simulação hidrológica com o modelo SWAT é realizada a partir da inserção de diferentes dados requeridos como entrada, além da adoção de uma sequência de procedimentos recomendada pelo modelo.

O SWAT requer quatro tipos principais de dados de entrada, três tipos são considerados dados espaciais e um tipo representa os dados tabulares. Os dados espaciais incluem o mapa de uso e ocupação do solo, o mapa de tipos de solo e o modelo digital de elevação (MDE) da área de estudo. Os dados tabulares utilizados pelo modelo SWAT para a modelagem hidrológica devem ser diários e de variáveis meteorológicas como: precipitação $(\mathrm{mm})$ (variável que mais influencia a modelagem com o SWAT), temperaturas máximas e mínimas do ar $\left({ }^{\circ} \mathrm{C}\right)$, umidade relativa do ar $(\%)$, velocidade do vento $\left(\mathrm{m} . \mathrm{s}^{-1}\right)$ e radiação solar $\left(\mathrm{MJ} . \mathrm{m}^{-}\right.$ $\left.{ }^{2}\right)$. A Tabela 1 apresenta as fontes de dados hidrológicos e meteorológicos utilizados para a modelagem hidrológica com o SWAT.

Tabela 1. Fontes dos dados hidrológicos e meteorológicos utilizados na modelagem hidrológica meteorológicos utilizados para a modelagem hidrológica com o SWAT.

\begin{tabular}{|c|c|c|}
\hline Descrição & Tipo de dado & Instituição / Agência \\
\hline $\begin{array}{l}\text { Mapa de uso e } \\
\text { ocupação do solo }\end{array}$ & Espacial & $\begin{array}{l}\text { Instituto Nacional de Pesquisas Espaciais (INPE); Ministério do } \\
\text { Meio Ambiente (MMA); Companhia Nacional de } \\
\text { Abastecimento (CONAB); Instituto Brasileiro de Geografia e } \\
\text { Estatística (IBGE) }\end{array}$ \\
\hline $\begin{array}{l}\text { Mapa de tipos de } \\
\text { solos }\end{array}$ & Espacial & $\begin{array}{l}\text { Empresa Brasileira de Pesquisa Agropecuária (EMBRAPA); } \\
\text { Artigos científicos }\end{array}$ \\
\hline $\begin{array}{l}\text { Modelo de Elevação } \\
\text { Digital (MDE) }\end{array}$ & Espacial & EMBRAPA Monitoramento por Satélite \\
\hline $\begin{array}{l}\text { Dados } \\
\text { pluviométricos }\end{array}$ & $\begin{array}{l}\text { Série de dados } \\
\text { diários }\end{array}$ & $\begin{array}{l}\text { Agência Nacional de Águas (ANA); Agência Pernambucana de } \\
\text { Águas e Clima (APAC) }\end{array}$ \\
\hline $\begin{array}{l}\text { Dados } \\
\text { meteorológicos }\end{array}$ & $\begin{array}{l}\text { Série de dados } \\
\text { diários }\end{array}$ & Instituto Nacional de Meteorologia (INMET) \\
\hline $\begin{array}{l}\text { Dados } \\
\text { fluviométricos }\end{array}$ & $\begin{array}{l}\text { Série de dados } \\
\text { diários }\end{array}$ & Agência Nacional de Águas (ANA) \\
\hline
\end{tabular}

O mapa de uso e ocupação do solo da subbacia do rio Mundaú foi elaborado a partir de ferramentas de processamento digital de imagens e de imagens do satélite LANDSAT 5, obtidas junto ao Instituto Nacional de Pesquisas Espaciais
(INPE). O mapeamento foi realizado empregandose o método de classificação supervisionada, adotando-se o classificador de máxima verossimilhança. $\mathrm{O}$ uso do solo foi dividido em cinco classes de interesse: i) pastagem, ii) 
agricultura, iii) caatinga, iv) área urbana, e v) água (Figura 2a).

Definidas as diferentes classes de uso do solo existentes na área em estudo, as mesmas foram reclassificadas de acordo com as classes disponíveis no banco de dados do SWAT. O modelo possui, dentre outras informações, um extenso banco de dados com múltiplos usos de solo e cobertura vegetal, com suas respectivas propriedades, que podem ser adotados em diferentes bacias hidrográficas. A associação do uso do solo com as classes existentes no banco de dados do SWAT, além da porcentagem de ocupação das classes na sub-bacia da BHRM são apresentadas na Tabela 2.

O mapa de tipos de solos (Figura $2 b$ ) foi obtido a partir de dados fornecidos pela Empresa Brasileira de Pesquisa Agropecuária (EMBRAPA), com base nas informações fornecidas pelo Zoneamento Agroecológicos de Pernambuco (ZAPE), o qual fornece mapas com escala de 1:100.000. Os solos existentes na bacia do rio Mundaú são, em maioria, Argissolos amarelos e Argissolos vermelho-amarelo; existem ainda Neossolos, Latossolos, Planossolos, Solos litólicos e Solos de mangue.

Tabela 2. Associação dos usos de solo existentes no estudo com os usos existentes no banco de dados do SWAT.

\begin{tabular}{cccc}
\hline Classe & Uso e ocupação do solo & Área (\%) & Classe do banco de dados do SWAT \\
\hline i & Pastagem & 89,86 & Pasture (PAST) \\
ii & Agricultura & 0,02 & Agricultural Land-Generic (AGRL) \\
iii & Caatinga & 9,40 & Range Brushes (RNGB) \\
iv & Área urbana & 0,58 & Residential (URBN) \\
v & Água & 0,14 & Water (WATR) \\
\hline
\end{tabular}

O modelo digital de elevação (MDE) foi obtido a partir da EMBRAPA Monitoramento por Satélite, onde são disponibilizados arquivos sobre a altimetria do Brasil. As imagens possuem formato Geotiff (16 bits), com resolução espacial de $90 \mathrm{~m}$ e Sistema de Coordenadas Geográficas WGS-84 (Figura 2c). (a)

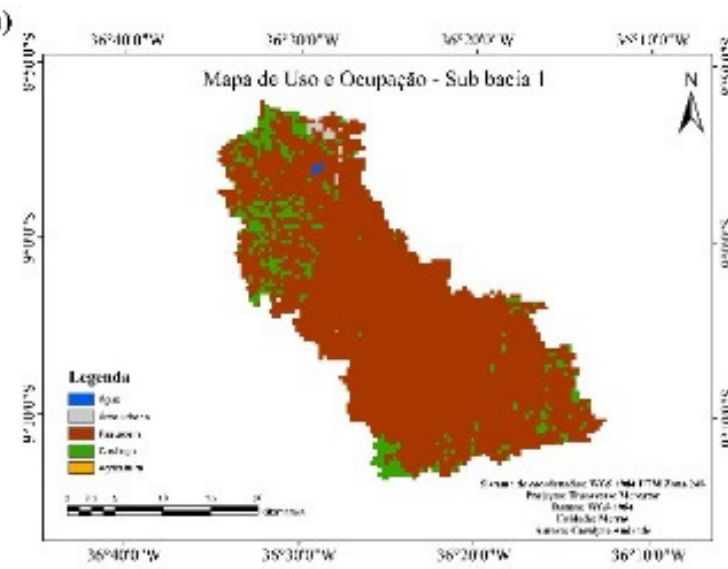

(c)

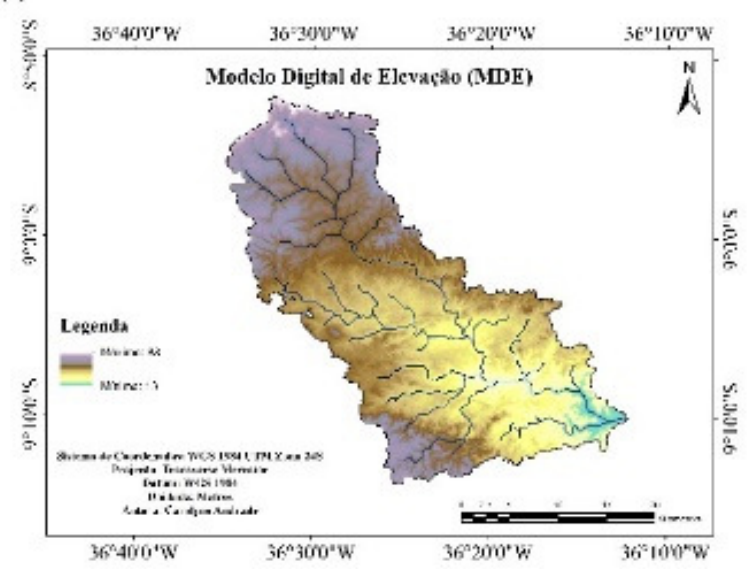

(b)

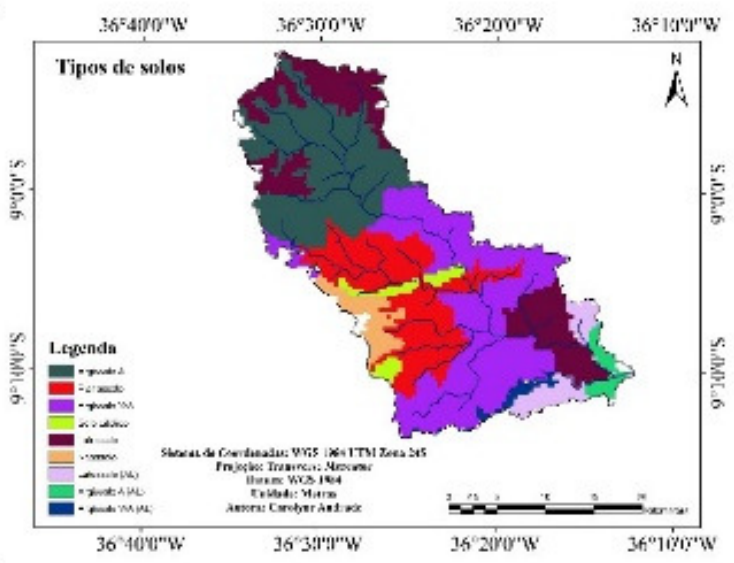

(d)

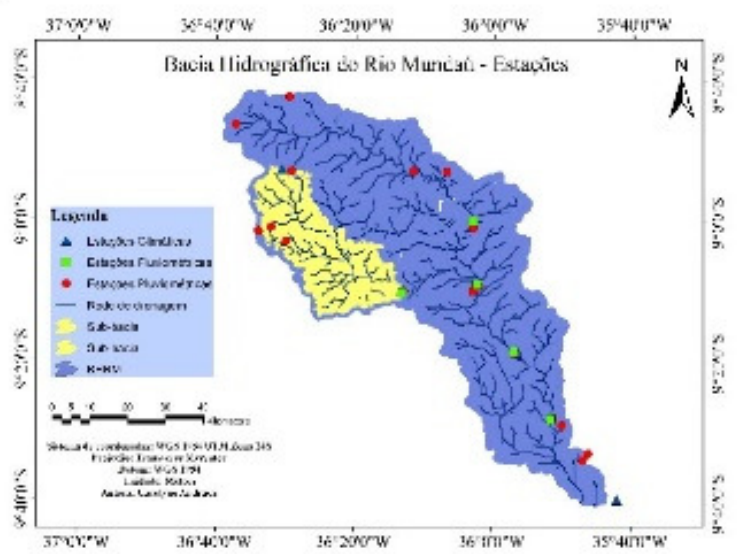

Figura 2. Mapas de uso e ocupação do solo (a), tipos de solos (b), modelo de elevação digital (c) e localização das estações (d) da sub-bacia do rio Mundaú. 
O levantamento dos dados hidrológicos foi realizado a partir das agências ANA e APAC. As estações utilizadas bem como suas localizações são apresentadas na Figura 2d. Foram selecionadas quatro estações pluviométricas, inseridas em dois municípios da bacia do Rio Mundaú e uma estação fluviométrica, inserida em um município alagoano. O levantamento dos dados meteorológicos foi realizado a partir do Instituto Nacional de Meteorologia (INMET), sendo utilizada a estação meteorológica localizada no município de Garanhuns-PE. As informações meteorológicas obtidas, necessárias para a modelagem com o SWAT, foram temperatura máxima e mínima $\left({ }^{\circ} \mathrm{C}\right)$, radiação solar $\left(\mathrm{MJ}^{-\mathrm{m}^{-2}}\right)$, umidade relativa $(\%) \mathrm{e}$ velocidade do vento $\left(\mathrm{m} \cdot \mathrm{s}^{-1}\right)$.

A série de dados tabulares considerada para a simulação hidrológica foi do período de 2000 a 2016 (17 anos), operando em passo de tempo diário, em que os dois primeiros anos (2000 e 2001) foram utilizados para o aquecimento do modelo SWAT, não sendo considerados na modelagem. Os valores simulados de vazão da subbacia de número 60 , correspondente ao exutório da área de estudo, foram comparados aos dados fluviométricos observados da estação Santana do Mundaú-AL.

Procedimento de calibração sistemática com o software SWATCUP

$\mathrm{O}$ procedimento de calibração anual da sub-bacia da BHRM foi realizado a partir do programa SWATCUP, um software independente, desenvolvido para análises de incertezas e sensibilidade, processos de calibração e validação, a partir de simulações com o SWAT (Abbaspour et al., 2007). O algoritmo SUFI-2 foi utilizado na calibração. A série de dados consideradas para a simulação hidrológica foi desde 2000 até o ano de 2016, sendo os dois primeiros anos destinados ao aquecimento do modelo e os anos de 2002 a 2009 para a calibração.

Para cada iteração foram observados a eficiência de índices estatísticos, como: o coeficiente de Nash-Sutcliffe (CNS), o percentual de tendência (PBIAS) e o coeficiente de determinação $\left(\mathrm{R}^{2}\right)$. Os métodos são calculados, respectivamente, através das Equações 6, 7 e 8:

$$
\begin{aligned}
& C N S=1-\frac{\sum_{i}\left(Q_{o b s}-Q_{s i m}\right)_{i}^{2}}{\sum_{i}\left(Q_{o b s, i}-\bar{Q}_{o b s}\right)^{2}} \\
& P B I A S=100 \frac{\sum_{i=1}^{n}\left(Q_{o b s}-Q_{s i m}\right)_{i}}{\sum_{i=1}^{n}\left(Q_{o b s, i}\right)} \\
& R^{2}=\frac{\left[\sum_{i}\left(Q_{o b s, i}-\bar{Q}_{o b s}\right)\left(Q_{s i m, i}-\bar{Q}_{s i m}\right)\right]^{2}}{\sum_{i}\left(Q_{o b s, i}-\bar{Q}_{o b s}\right)^{2} \sum_{i}\left(Q_{s i m, i}-\bar{Q}_{s i m}\right)^{2}}
\end{aligned}
$$

onde $\mathrm{Q}_{\text {obs }}$ é vazão observada, $\mathrm{Q}_{\text {sim }}$ é vazão simulada, $\bar{Q}_{o b s}$ é a média das vazões observadas e $\bar{Q}_{\text {sim }}$ é a média das vazões simuladas.

$\mathrm{O}$ CNS varia entre menos infinito e 1 (valor ótimo). Valores entre 0 e 1 geralmente são vistos como níveis de desempenho aceitáveis e valores $\leq 0.0$, indicam que é melhor utilizar a média dos dados observados que o valor predito pelo modelo (Nash \& Sutcliffe, 1970). Em relação ao PBIAS, seu valor ótimo é de 0 , baixas magnitudes indicam boa precisão na simulação do modelo. Valores positivos indicam tendência percentual do modelo de subestimação e valores negativos, tendência percentual de superestimação (Gupta et al., 1999). O valor ótimo para o coeficiente de determinação $\left(\mathrm{R}^{2}\right)$ é igual a 1 , por isso, quanto mais próximo da unidade, melhor será o ajuste entre os dados observados e simulados.

O desempenho do modelo SWAT a partir dos valores dos índices estatísticos CNS e PBIAS é apresentado na Tabela 3.

Tabela 3. Desempenho do modelo SWAT. Fonte Santos (2013). CNS = coeficiente de Nash-Sutcliffe; PBIAS $=$ percentual de tendência.

\begin{tabular}{lcc}
\hline Desempenho do modelo SWAT & CNS & PBIAS \\
\hline Muito bom & $0,75<\mathrm{CNS} \leq 1,00$ & PBIAS $< \pm 10$ \\
Bom & $0,65<\mathrm{CNS} \leq 0,75$ & $\pm 10 \leq$ PBIAS $< \pm 15$ \\
Satisfatório & $0,50<\mathrm{CNS} \leq 0,65$ & $\pm 15 \leq$ PBIAS $< \pm 25$ \\
Insatisfatório & $\mathrm{CNS} \leq 0,50$ & $\pm 25 \geq$ PBIAS \\
\hline
\end{tabular}

\section{Resultados}

A porção estudada $\left(754,99 \mathrm{~km}^{2}\right)$ da bacia hidrográfica do rio Mundaú $\left(4.126 \mathrm{~km}^{2}\right)$ foi dividida pelo modelo SWAT em 68 sub-bacias (o exutório corresponde a sub-bacia 60), com áreas variando entre 0,04 e $37,08 \mathrm{~km}^{2}$.

A partir do dado de entrada MDE (Figura $3 b$ ), que possui um valor máximo de $63 \mathrm{~m}$ (porção norte da sub-bacia) e um valor mínimo de $13 \mathrm{~m}$ (porção sul da sub-bacia), o modelo SWAT gerou o mapa de declividade, onde a maior parte apresenta declividade de 0 a $2 \%$ e pequenas áreas, principalmente na porção sul, apresentam declividades acima de $2 \%$. 
No relatório sobre as HRU's, onde são gerados o número total de microbacias, o número de HRU's resultou em 199.

A Tabela 4 apresenta o resultado dos processos hidrológicos simulados pelo modelo SWAT. A ilustração desses processos é apresentada na Figura 3.
A precipitação anual média da BHRM foi igual a $1.075 \mathrm{~mm}$, a evapotranspiração potencial ou de referência média anual foi igual a $1.262,8 \mathrm{~mm}$, a ascensão do aquífero raso anual média foi de $25,26 \mathrm{~mm}$, sendo que o escoamento superficial anual médio foi igual a $132,25 \mathrm{~mm}$.

Tabela 4. Processos hidrológicos simulados pelo SWAT para a sub-bacia da Bacia Hidrográfica do Rio Mundaú-BHRM.

\begin{tabular}{lc}
\hline Processo hidrológico & Sub-bacia 60 (exutório) \\
\hline Escoamento superficial & 132,25 \\
Precipitação & 1.075 \\
Evapotranspiração & 674,2 \\
Evapotranspiração potencial & $1.262,8$ \\
Percolação para o aquífero raso & 268,85 \\
Ascensão do aquífero raso & 25,26 \\
Fluxo lateral & 1,13 \\
Curva-Número & 76,64 \\
Recarga para o aquífero profundo & 13,44
\end{tabular}

* Todas as unidades em mm.

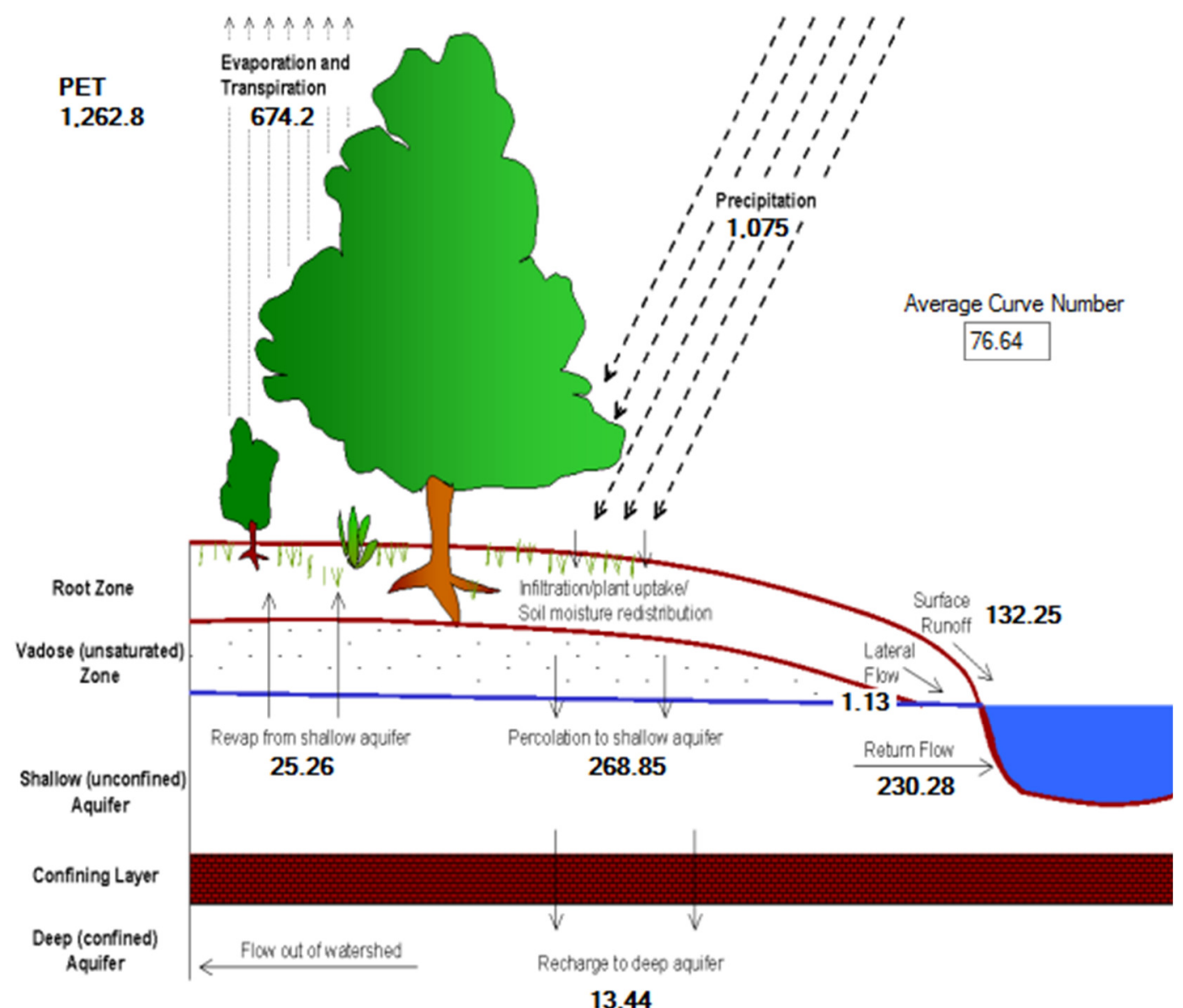

Todas as unidades em $\mathrm{mm}$

Figura 3. Representação dos processos simulados pelo modelo SWAT para a sub-bacia da Bacia Hidrográfica do Rio Mundaú-BHRM.

A evapotranspiração real anual média resultou em $674,2 \mathrm{~mm}$.
A Figura 4 apresenta o hidrograma de vazão mensal observado e simulado pelo modelo 
SWAT no período de calibração (2002-2009) e o hietograma de precipitação mensal na sub-bacia

Analisando os dados de vazão, o valor médio mensal observado da seção de monitoramento Santana do Mundaú-AL para o período da calibração foi igual a $7,3 \mathrm{~m}^{3} \cdot \mathrm{s}^{-1}$, enquanto que o valor médio dos dados simulados pelo modelo SWAT foi $10,3 \mathrm{~m}^{3} . \mathrm{s}^{-1}$. O escoamento superficial médio simulado pelo modelo foi igual a $32 \mathrm{~mm}$.

\section{Calibração (2002-2009)}

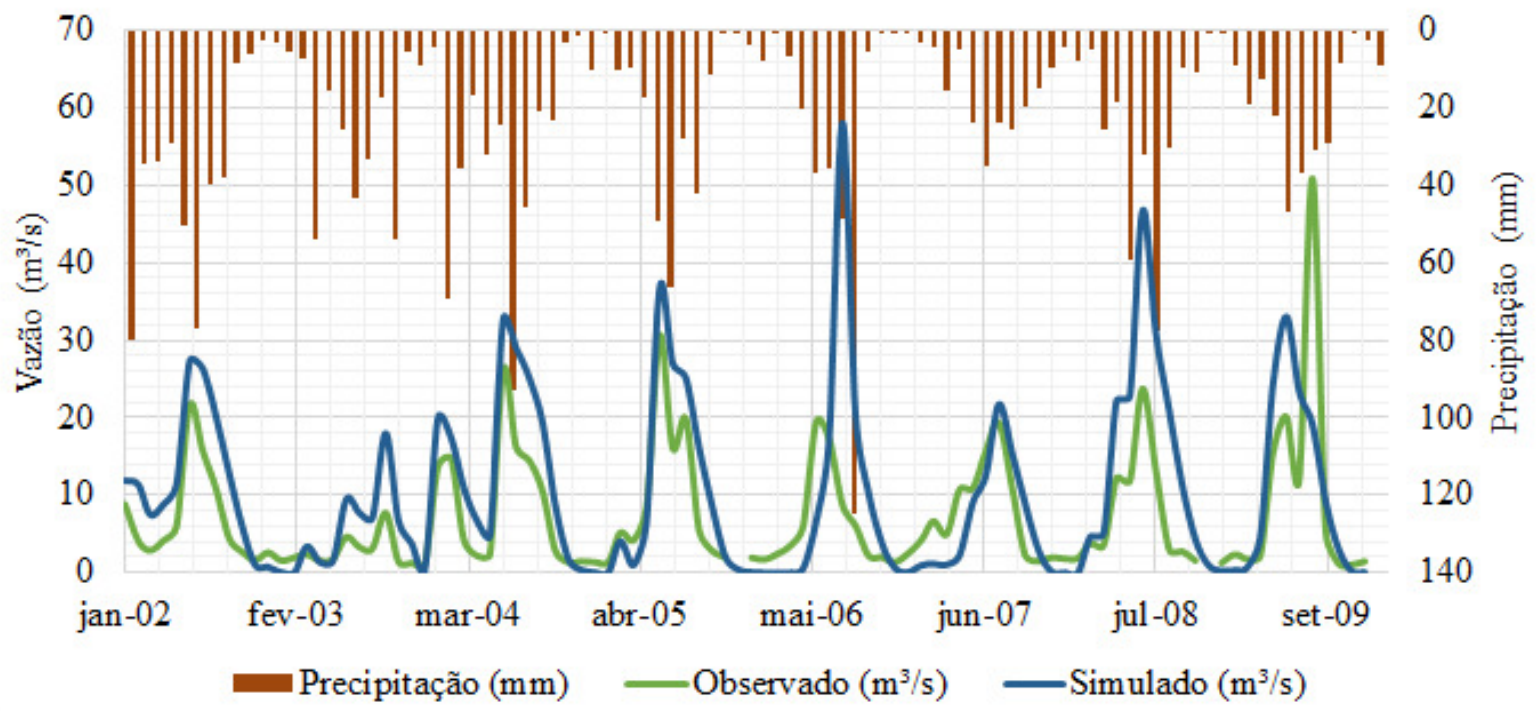

Figura 4. Hidrograma observado e simulado mensais para a BHRM no período de calibração (2002-2009) e hietograma de precipitação mensal na sub-bacia.

A Tabela 5 apresenta os parâmetros utilizados para a calibração do modelo SWAT na sub-bacia da BHRM. Tais parâmetros foram selecionados a partir de estudos e análises de sensibilidades realizadas anteriormente na BHRM, as quais estão descritas em Andrade et al. (2017).
A Tabela 6 apresenta o resultado dos índices estatísticos utilizados para avaliar o desempenho do modelo SWAT na sub-bacia da BHRM na fase de calibração.

Tabela 5. Parâmetros utilizados na calibração do modelo SWAT, a partir dos dados fluviométricos da subbacia da Bacia Hidrográfica do Rio Mundaú-BHRM.

\begin{tabular}{lccc}
\hline Parâmetro & Intervalo de variação & Valor calibrado \\
\hline r_CN2.mgt & Valor mínimo & Valor máximo & 0,20 \\
v__RCHRG_DP.gw & 0,05 & 0,201 & 0,13 \\
v__GWQMN.gw & 0,01 & 5894,47 & 0,01 \\
v_ALPHA_BF.gw & 3763,03 & 0,02 & 5721,82 \\
r_SOL_AWC().sol & 0,00 & 0,21 & 0,01 \\
v_ESCO.hru & $-0,03$ & 0,74 & $-0,03$ \\
r_SOL_K().sol & 0,58 & 0,16 & 0,59 \\
v_GW_DELAY.gw & $-0,30$ & 127,52 & 0,13 \\
v_GW_REVAP.gw & 29,40 & 0,10 & 90,14 \\
v__SHALLST.gw & 0,08 & 1354,23 & 0,09 \\
v_SURLAG.bsn & 984,88 & 4,71 & 1308,06 \\
v__LAT_TTIME.hru & 0,36 & 1,91 & 3,75 \\
r_SOL_Z().sol & 1,26 & 0,05 & 1,47 \\
v_CH_N2.rte & $-0,03$ & 0,11 & $-0,02$ \\
v_CH_K2.rte & 0,07 & 17,68 & 0,07 \\
r_OV_N.hru & 10,55 & 0,11 & 16,69 \\
\hline
\end{tabular}


Tabela 6. Índices estatísticos resultantes da etapa de calibração do modelo SWAT na sub-bacia da Bacia Hidrográfica do Rio Mundaú-BHRM.

\begin{tabular}{lcc}
\hline Índice Estatístico & Valor & Desempenho \\
\hline CNS & 0,53 & Satisfatório \\
PBIAS & $-7,1$ & Muito Bom \\
$\mathrm{R}^{2}$ & 0,56 & - \\
\hline
\end{tabular}

O CNS resultou em 0,53 para o período de calibração. Em relação ao PBIAS, o valor encontrado foi de $-7,1 \%$ para a calibração. $O$ valor para o coeficiente de determinação $\left(R^{2}\right)$ foi de 0,56 para a calibração.

Considerando os resultados de CNS e PBIAS, pode-se dizer que o modelo SWAT teve desempenhos satisfatório e muito bom, respectivamente, na modelagem hidrológica da sub-bacia do rio Mundaú para o período de calibração.

\section{Discussão}

Cada área subdividida pelo modelo não deve ultrapassar $100 \mathrm{~km}^{2}$, uma vez que áreas maiores do que esse valor, podem sofrer efeitos de escala, ou seja, diferentes usos e tipos de solo, alterações atmosféricas, diferenças na rede de drenagem, resultando em processos hidrológicos com comportamentos distintos (Krysanova et al., 1998). Abbaspour et al. (2015) discretizaram o continente europeu em 8.592 sub-bacias. Bressiani et al. (2015) obtiveram 232 sub-bacias na bacia hidrográfica do rio Jaguaribe $\left(73.000 \mathrm{~km}^{2}\right)$, com uma área média de $315 \mathrm{~km}^{2}$. Apesar de a área discretizada por esses autores representar um valor superior a $100 \mathrm{~km}^{2}$ (recomendado como limite máximo), as bacias trabalhadas possuem áreas de dimensões muito elevadas, por isso, os efeitos de escala são reduzidos e a discretização áreas pode ser aumentada, sem comprometer os resultados da modelagem hidrológica. De fato, os autores encontraram valores de CNS de até 0,79.

Os mapas de tipos e uso do solo juntamente com o mapa de declividade, gerado anteriormente, fornecem as informações necessárias para que o modelo delimite as Unidades de Resposta Hidrológica (HRU's) (Winchell et al., 2010).

As HRU's correspondem a áreas com combinações de uso do solo, tipos de solos e classes de declividade semelhantes. Abbaspour et al. (2015) discretizou o continente europeu em 60.012 HRU's. Bressiani et al. (2015) obteve 1.145 HRU's.

Na modelagem com o SWAT, são gerados diversos resultados que simulam o comportamento das varáveis hidrossedimentólogicas ao longo do tempo. As informações incluem precipitação, evapotranspiração, escoamento superficial, fluxo de base, ascensão capilar, percolação para o aquífero raso, recarga para o aquífero profundo, informações sobre uso do solo, manejo, pesticidas, nutrientes, sedimentos, entre outros.

Em relação a evapotranspiração real (ET) os resultados do modelo SWAT se aproximam dos resultados de Gondim et al. (2015), que mediram a ET em área de pastagem na mesma bacia hidrográfica e encontraram um valor anual de $543,8 \mathrm{~mm}$.

Houve uma superestimação da vazão pelo modelo em $41 \%$. É possível notar que os picos de vazão observados são acompanhados pelo SWAT.

O CNS está dentro do intervalo encontrado por vários pesquisadores. Diversos autores utilizaram o CNS como índice de desempenho relacionado à resposta do comportamento de vazões em bacias hidrográficas simuladas com o SWAT. Quyen et al. (2014) utilizando o modelo SWAT para simular a vazão da bacia hidrográfica Srepok, no Vietnã, e observar os impactos das alterações do uso do solo no escoamento da bacia, obtiveram resultados de CNS de 0,75 na calibração (1981-200) e 0,55 na validação (2001-2009). Xue et al. (2014), analisando a geração de vazão e sedimentos na bacia Huolin, na China, encontraram valores de CNS de 0,83 para o período de calibração (1991-1996) e de 0,92 para o período de validação (1997-2000). Abbaspour et al. (2015) aplicando o modelo SWAT para países do continente europeu encontraram valores de CNS no processo de calibração que variaram desde 0,03 (Polônia) até 0,91 (Rússia). Bressiani et al. (2015) aplicando o modelo SWAT para analisar as respostas hidrológicas a partir de diferentes fontes, escalas espaciais e resoluções temporais de dados de entrada climáticos na bacia hidrográfica do rio Jaguaribe $\left(73.000 \mathrm{~km}^{2}\right)$, Nordeste do Brasil, encontraram valores de CNS desde 0,32 até 0,79. Creech et al. (2015), estudando os impactos antrópicos relacionados a produção de sedimentos sobre a bacia do rio São Francisco $\left(630.000 \mathrm{~km}^{2}\right)$, localizada nos Estados de Minas Gerais, Bahia, Pernambuco, Alagoas e Sergipe (com uma pequena porção em Goiás e no Distrito Federal), encontraram valores de CNS variando desde 0,42 (rio Paraopeba) até 0,75 (rio São Francisco) para o período de calibração (1996-2005) e valores desde 0,42 (rio Pará) até 0,77 (rio São Francisco) para o período de validação (2006-2010). Lin et al. (2015) analisando os impactos das mudanças do uso do solo no escoamento da bacia hidrográfica do rio Jinjiang na China, utilizando o modelo SWAT a nível mensal, encontraram melhores valores de CNS para o período de calibração (2002-2007) de 0,97 e para o período de validação (2008-2010) também de 0,97. Neupane et al. (2015) projetando 
mudanças hidrológicas associadas às alterações climáticas e de uso do solo em uma bacia montanhosa do Himalaia, utilizando o modelo SWAT, obtiveram melhores resultados de CNS de 0,86 e 0,89 durante as etapas de calibração e validação, respectivamente. Silva et al. (2016), estudando os efeitos de cenários de uso do solo na vazão e produção de sedimentos na bacia do Rio Cobres em Portugal, obtiveram resultados de CNS de 0,63 na calibração e 0,57 na validação. Kumar et al. (2017) realizando calibração e análises de incertezas para a predição da vazão na bacia do rio Tons $\left(18,318 \mathrm{~km}^{2}\right)$, Índia, encontraram CNS de 0,73 durante a calibração.

Bressiani et al. (2015) encontraram variações, em termos absolutos, do PBIAS (\%) desde 58,21 $(-58,21)$ até $6,75(-6,75)$. Lin et al. (2015) encontraram melhores valores de PBIAS (\%) para o período de calibração (2002-2007) de 3,8 e para o período de validação $(2008-2010)$ de 4,73. Neupane et al. (2015) obtiveram melhores resultados de PBIAS (\%) de 2,5 e -0,9 durante as etapas de calibração e validação, respectivamente. Kumar et al. (2017) encontraram PBIAS (\%) de 3,55 durante a calibração.

Quyen et al. (2014) obtiveram resultados de $\mathrm{R}^{2}$ de 0,83 na calibração (1981-200) e 0,72 na validação (2001-2009). Xue et al. (2014) encontraram valores de CNS de 0,85 para o período de calibração (1991-1996) e de 0,93 para o período de validação (1997-2000). Abbaspour et al. (2015) encontraram valores de $\mathrm{R}^{2}$ no processo de calibração que variaram desde 0,2 (Hungria) até 0,78 (Portugal). Lin et al. (2015) encontraram melhores valores de $\mathrm{R}^{2}$ para o período de calibração (2002-2007) de 0,97 e para o período de validação (2008-2010) de 0,98. Kumar et al. (2017) encontraram $R^{2}$ de 0,74 durante a calibração. Silva et al. (2016) obtiveram resultados de $\mathrm{R}^{2}$ de 0,81 na calibração e 0,83 na validação.

\section{Conclusão}

Foi possível realizar a calibração com o modelo SWAT na sub-bacia do rio Mundaú, obtendo-se desempenhos satisfatório (CNS) e muito bom (PBIAS). Com o modelo calibrado, estudos futuros podem ser realizados com o SWAT, a fim de validá-lo e prever possíveis impactos de mudanças climáticas e de uso do solo na bacia estudada. Estudos como este, assim como tantos outros estudos hidrológicos, têm mostrado que, cada vez mais, os dados experimentais são imprescindíveis para a adequada representação e entendimento dos processos através da modelagem hidrológica.

\section{Agradecimentos}

Os autores agradecem à Fundação de Amparo à Ciência e Tecnologia de PernambucoFACEPE, pela bolsa de doutorado do primeiro autor (IBPG-0231-5.03/14 e BCT-0176-5.03/14 convênio APAC), ao CNPq pela Bolsa PQ do segundo, terceiro e quarto autores, aos Projetos PVE-CAPES (PVE aupex 2792/2013 proc. 23038007733/2013-76, ao Conselho Nacional de Desenvolvimento Científico e Tecnológico-CNPq (Processo N. 446254/2015-0), ao Projeto REHIDRO/FINEP, CNPq (Processo N. 446254/2015-0) e ao Projeto do Edital Universal do CNPq (448236/2014-1).

\section{Referências}

ABBASPOUR, K. C.; YANG, J.; MAXIMOV, I.; SIBER, R.; BOGNER, K.; MIELEITNER, J.; ZOBRIST, J.; SRINIVASAN, R. 2007. Modelling hydrology and water quality in the prealpine/alpine Thur watershed using SWAT. Journal of Hydrology, v. 333, p. 413-430.

ABBASPOUR, K. C.; ROUHOLAHNEJAD, E.; VAGHEFI, S.; SRINIVASAN, R.; YANG, H.; KLOVE, B. 2015. A continental-scale hydrology and water quality model for Europe: Calibration and uncertainty of a high-resolution large-scale SWAT model. Journal of Hydrology, v. 524, p. 733-752.

ANDRADE, C. W. L.; MONTENEGRO, S. M. G. L.; LIMA, J. R. S.; MONTENEGRO, A. A. A.; SRINIVASAN, R. 2017. Análise de sensibilidade de parâmetros do modelo SWAT em uma subbacia da região Nordeste, Brasil. Revista Brasileira de Geografia Física, v. 10, n. 2, p. 440-453.

ARNOLD, J. G.; KINIRY, J. R.; SRINIVASAN, R.; WILLIAMS, J. R.; HANEY, E. B.; NEITSCH, S. L. 2012. Input/Output Documentation version 2012. Texas Water Resources Institute - TR-439, $650 \mathrm{p}$.

BRESSIANI, D. A. 2016. Coping with hydrological risks through flooding risk index, complex watershed modeling, different calibration techniques, and ensemble streamflow forecasting. Tese de Doutorado, Escola de Engenharia de São Carlos - Universidade de São Paulo, São Carlos, São Paulo, Brasil. 192p.

BRESSIANI, D. A.; SRINIVASAN, R.; JONES, C. A.; MENDIONDO, E. M. 2015. Effects of spatial and temporal weather data resolutions on streamflow modeling of a semi-arid basin, Northeast Brazil, International Journal of 
Agricultural and Biological Engineering, v. 8, n. 3, p. $125-139$.

CREECH, C. T.; SIQUEIRA, R. B.; SELEGEAN, J. P.; MILLER, C. 2015. Anthropogenic impacts to the sediment budget of São Francisco River navigation channel using SWAT. International Journal of Agricultural and Biological Engineering, v. 8, n. 3, p. 140-157.

GONDIM, P. S. S.; LIMA, J. R. S.; ANTONINO, A. C. D.; HAMMECKER, C.; SILVA, R. A. B.; GOMES, C. A. 2015. Environmental control on water vapour and energy exchanges over grasslands in semiarid region of Brazil. Revista Brasileira de Engenharia Agrícola e Ambiental, v. 19, p. 3-8.

GUPTA, H. V.; SOROOSHIAN, S.; YAPO, P. O. 1999. Status of automatic calibration for hydrologic models: Comparison with multilevel expert calibration. Journal Hydrologic Engineering, v. 4, n. 2, p. 135-143.

KRYSANOVA, V.; MÜLLER-WOHLFEIL, D. I.; BECKER, A. 1998. Development and test of a spatially distributed hydrological/water quality model for mesoscale watersheds, Ecological Modelling, v. 106, n. 2, p. 261-289.

KUMAR, N.; SINGH, S. K.; SRIVASTAVA, P. K.; NARSIMLU, B. 2017. SWAT Model calibration and uncertainty analysis for streamflow prediction of the Tons River Basin, India, using Sequential Uncertainty Fitting (SUFI-2) algorithm. Modeling Earth Systems and Environment, v. 3, n. $30,13 p$.

LEAL, I. R.; SILVA, J. M. C.; TABARELLI, M.; LACHER JUNIOR, T. E. 2005. Changing the course of biodiversity conservation in the Caatinga of Northeastern Brazil. Conservation Biology, v. 19, n. 3, p. 701-706.

LIN, B.; CHEN, X.; YAO, H.; CHEN, Y.; LIU, M.; GAO, L.; JAMES, A. 2015. Analyses of land use change impacts on catchment runoff using different time indicators based on SWAT model. Ecological Indicators, v. 58, p. 55-63.

MONTENEGRO, S. M. G. L.; SILVA, B. B.; ANTONINO, A. C. D.; LIMA, J. R. S.; SOUZA, E. S.; OLIVEIRA, L. M. M.; MOURA, A. E. S. S.; SOUZA, R. M. S. 2014. Hydrological studies in experimental and representative basins in Pernambuco State, Brazil. Evolving Water Resources Systems: Understanding, Predicting and
Managing Water-Society Interactions Proceedings of ICWRS 2014, Bolonha, Itália. Publ. 364p.

NASH, J. E.; SUTCLIFFE, J. V. 1970. River flow forecasting through conceptual models: Part 1. A discussion of principles. Journal of Hydrology, $\mathrm{v}$. 10 n. 3, p. 282-290.

NEITSCH, S. L.; ARNOLD, J. G.; KINIRY, J. R.; WILLIAMS, J. R. 2005. Soil and water assessment tool: Theoretical documentation version 2005. Temple: Blackland Research Center. 541p.

NEUPANE, R. P.; WHITE, J. D.; ALEXANDER, S. E. 2015. Projected hydrologic changes in monsoon-dominated Himalaya Mountain basins with changing climate and deforestation. Journal of Hydrology, v. 525, p. 216-230.

PAUWELS, G. J. 2002. Atlas Geográfico Melhoramentos, $5^{\text {a }}$ Ed., Editora Melhoramentos, São Paulo.

QUADRO, M. F. L.; MACHADO, L. H. R.; CALBETE, S.; BATISTA, N. N. M.; SAMPAIO, G. 1996. Climatologia, precipitação e temperatura no período de 1986 a 1996. Revista Climanálise, Edição comemorativa de 10 anos, CPTEC/INPE.

QUYEN, N. T. N.; LIEM, N. D.; LOI, N. K. 2014. Effect of land use change on water discharge in Srepok watershed, Central Highland, Viet Nam. International Soil and Water Conservation Research, v. 2, n. 3, p. 74-86.

RALLISON, R. E.; MILLER, N. 1981. Past, present and future SCS runoff procedure. In: SINGH, V. P. (Ed.). Rainfall runoff relationship. Littleton, USA: Water Resources Publication, pp. 353-364.

HARGREAVES, G. H.; SAMANI, Z. A. 1985. Reference crop evapotranspiration from temperature. Applied Engeneering Agriculture, v. 1, n. 1, p. 96-99.

SILVA, R. M.; SANTOS, C. A. G.; SILVA, V. C. L.; MEDEIROS, I. C.; MOREIRA, M.; CORTEREAL, J. 2016. Efeitos de cenários de uso do solo na vazão e produção de sedimentos na Bacia do Rio Cobres, Portugal. Geociências, v. 35, n. 4, p. 609622.

WERNECK, F. P. 2011. The diversification of eastern South American open vegetation biomes: Historical biogeography and perspectives. 
Journal of Environmental Analysis and Progress V. 02 N. 03 (2017) 227-238

Quaternary Science Reviews, v. 30, n. 13-14, p. 1630-1648.

WINCHELL, M.; SRINIVASAN, R.; DI LUZIO, M.; ARNOLD, J. G. 2010. ArcSWAT interface for SWAT2009 User's guide. USDA Agricultural
Research Service and Texas A\&M Blackland Research Center, Temple, Texas. 431p.

XUE, C.; MASCE, B. C.; WU, H. 2014. Parameter uncertainty analysis of surface flow and sediment yield in the Huolin Basin, China. Journal of Hydrologic Engineering, v. 19, p. 1224-1236. 